\title{
"What you see may not be what you get": Reverse contingency and perceived loss aversion in pigeons
}

\author{
Jacelyn Sturgill ${ }^{1} \cdot$ Cameron Bergeron $^{1} \cdot$ Thomas Ransdell $^{1} \cdot$ Tatum Colvin $^{1} \cdot$ Gia Joshi $^{1} \cdot$ Thomas R. Zentall $^{1}$ (D)
}

Accepted: 21 December 2020 / Published online: 2 February 2021

(C) The Psychonomic Society, Inc. 2021

\begin{abstract}
The reverse-contingency task is a task in which one is given a choice between two rewards, but one receives the larger amount only if one chooses the smaller amount. This task is very difficult for chimpanzees unless the choice is between symbolic representations of the amounts. We found that pigeons can learn this task easily, if the reward amounts are associated with distinctive colors and the choice is delayed by $5 \mathrm{~s}$. The reverse-contingency task involves three components: a loss when choosing one alternative, a gain when choosing the other, and the contrast between what was expected and what occurred. In Experiment 2 we separated the loss from the gain and found that experiencing a loss is sufficient for pigeons to learn to avoid that alternative. Finally, we found evidence for perceived loss aversion. When pigeons were offered a small amount of food and they received that amount, they preferred it over an alternative that offered them a larger amount but gave them only the smaller amount (a perceived loss). The results indicate that loss aversion, based on reference dependence, is likely a general phenomenon, and not only found in humans and other primates. We suggest that it can be attributed to contrast, the difference between what is expected and what is obtained, and it is related to the endowment effect and the mere ownership effect found in humans.
\end{abstract}

Keywords Loss aversion $\cdot$ Reverse contingency $\cdot$ Reference dependence $\cdot$ Contrast $\cdot$ Pigeons

\section{Introduction}

Theories of learning are often based on the discrepancy between expected and actual outcomes (Bush \& Mosteller, 1951). The greater the discrepancy between reward expectation and outcome, the greater learning will occur. A physiological model of this discrepancy, referred to as reward-prediction error, and based on dopamine release, has been suggested by Mirenowicz and Schultz (1994) and others (Montague, Dayan, \& Sejnowski, 1996; Schultz, Dayan, \& Montague, 1997).

In humans, the general category of effects that are influenced by the discrepancy between expectation and occurrence is often referred to as reference dependence (Kahneman \& Tversky, 1979). Reference dependence is thought to be responsible for many contrast effects (the difference between outcomes expected and those obtained) including the greater

Thomas R. Zentall

zentall@uky.edu

1 Department of Psychology, University of Kentucky, Lexington, KY 40506-0044, USA aversion that humans have to incurring a loss than would be expected by utility theory (von Neumann \& Morgenstern, 1944). That is, for humans, the disutility of giving up an object is greater than the utility associated with acquiring it.

To what extent is this bias to avoid losses a cultural phenomenon based on the human desire to own things? Humans tend to value things that belong to them more than things that do not (the mere ownership or endowment effect; Kahneman, Knetsch, \& Thaler, 1990; Weaver \& Fredrick, 2012), an effect that is inconsistent with standard economic theory. The fact that this effect is assumed not to vary very much over time or between cultures (Becker, 1962) suggests that it may have an evolutionary basis. In support of this hypothesis, Chen, Lakshminarayanan, and Santos (2006) found that capuchin monkeys, too, show evidence of reference dependence. In one of their experiments, they offered monkeys a choice between giving a token to (1) an experimenter who offered them two pieces of apple but gave them only one and (2) an experimenter who offered them one piece of apple and gave them the piece of apple. Although the net result of the choice was the same, the monkeys showed a strong preference for the experimenter who did not remove one of the shown pieces of apple. 
Chen et al. (2006) proposed that both rats and pigeons are different from monkeys because those species appear to obey the laws of demand (Kagel et al., 1975). Referring to those other species, Chen et al. argued that "their limited cognitive abilities make it difficult to investigate more subtle aspects of economic choice, including many important and systematic human biases" (p. 519). The procedure used by Chen et al. did involve a complex procedure involving an exchange of tokens for reward between two experimenters. In principle, however, experiments assessing similar choice procedures could be conducted with pigeons.

Imagine that a pigeon is offered a choice between two options, one alternative would present the pigeon with two pellets of food but if it chooses that option it would get only one pellet. The other alternative would present the pigeon with one pellet and if it chooses that option it would get the one pellet. Three reasonable hypotheses can be generated. First, according to Chen et al. (2006), the pigeon might be indifferent with regard to the two alternatives because the net result would be one pellet in both cases. Second, the pigeon may respond based on what it is shown; two pellets shown is better than one pellet shown (what you see is what you might get). Finally, the pigeon may be averse to losing the second pellet and may prefer the reliable one pellet instead (reference dependence).

The purpose of the present experiments was to investigate reference dependence in pigeons under conditions similar to those studied by Chen et al. (2006), but without the token exchange or the two experimenters. We first established that pigeons could discriminate between the offers of one versus two pellets. We then tested them under conditions of actual loss, but one compensated for by an actual gain (Experiment 1), a reverse contingency task that was found to be very difficult for chimpanzees (Boysen \& Berntson, 1995). The reverse contingency experiment confounds an actual loss with an actual gain. We then tested them for their sensitivity to an actual loss; however, the pigeons had to discriminate between being offered two pellets but receiving only one pellet and being offered two pellets and receiving two pellets (Experiment 2). Finally, we tested the pigeons for a perceived loss by offering them two pellets but giving them only one versus offering them one pellet and giving them one (Experiment 3).

\section{General methods}

\section{Subjects}

Eight unsexed White Carneau pigeons (5-12 years of age), purchased from the Palmetto Pigeon Plant, Sumter, SC, USA served as subjects. All the pigeons had had experience with simultaneous and successive color discriminations. Each pigeon was housed in an individual wire cage $(28 \times 38 \times 30.5$ $\mathrm{cm})$. Throughout the study, the pigeons were maintained at $85 \%$ of their free-feeding body weight with free access to water and grit on a 12:12-h light:dark cycle. The pigeons were maintained in accordance with a protocol approved by the Institutional Animal Care and Use Committee at the University of Kentucky.

\section{Apparatus}

The apparatus was made of wood painted red on one side, green on the other (see Fig. 1). On each end of the apparatus were four $2.5-\mathrm{cm}$ diameter holes $(0.6 \mathrm{~cm}$ deep) spaced $1.0 \mathrm{~cm}$ apart, two on each end of the red side and two on each end of the green side. The apparatus could be turned such that the red side could be either on the right or on the left (arrow in Fig. 1). There were also four slides, each of which could cover or uncover one of the holes in front of the pigeon.
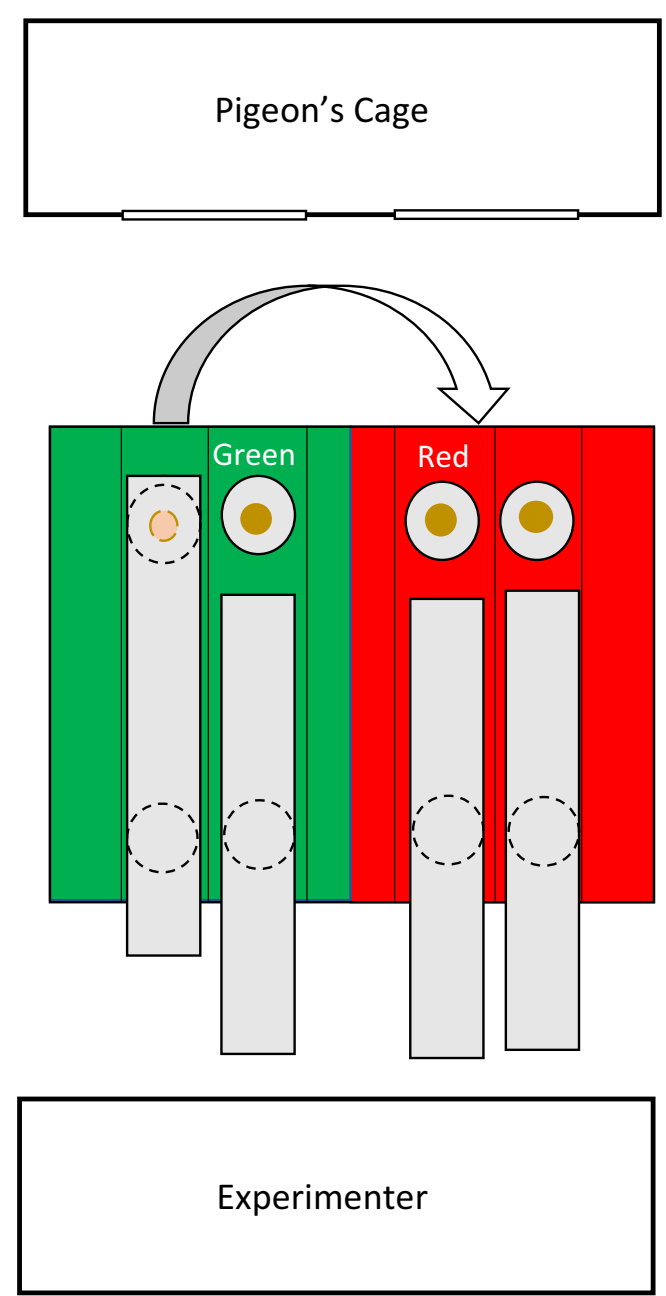

Fig. 1 Top view of the apparatus used in the experiment. Slides could cover or uncover shallow holes into which pellets were placed. The apparatus could be turned $180^{\circ}$ so that the red side would appear on the left and the green side on the right. The apparatus was baited in this position and then moved closer to the pigeon's cage 


\section{Procedure}

Each pigeon's cage had two openings on the front that allowed it to be tested in its home cage, which was moved to the testing room. In the testing room, the experimenter sat facing the pigeon with the apparatus between them. On each trial the front edge of the apparatus was placed about $30 \mathrm{~cm}$ from the front of the pigeon's cage, where it was baited with pellets. The apparatus was moved to the front of the pigeon's cage $5 \mathrm{~s}$ after baiting. When the pigeons see and hear the pellets being placed in the holes of the apparatus, they become very excited. They flap their wings and we have found that they tend to choose impulsively (Zentall, Case, \& Berry, 2017; Zentall \& Raley, 2019). The delay between baiting and choice was used because we have found that such a delay reduced impulsive choice by pigeons.

Through one of the two holes in the front of its cage, the pigeon could choose either the red or the green side of the apparatus. Once the pigeon made its choice and removed the pellet from the hole(s), the apparatus was pulled back toward the experimenter. If the next trial called for it, the apparatus was turned $180^{\circ}$ and was rebaited. The intertrial interval was 5 s. All sessions consisted of 32 trials. Sessions were conducted 6 days a week.

Pretraining All pigeons were initially tested for their ability to discriminate one pellet from two pellets with redundant color cues. The cues associated with the one- and two-pellet alternatives were reversed to determine if the colors controlled choice and to demonstrate that the pigeons could learn that the two pellets were now on the side of the other color. When the colors were reversed, the pigeons showed strong control by the colors until they learned the reversal.

\section{Experiment 1}

The procedure used in Experiment 1 involved three different principles of interest. First, as in pretraining, the outcome of the choice always involved one alternative with a better outcome than the other. Second, the choice of the alternative that presented two pellets involved the removal of one pellet - a loss. Finally, unlike pretraining, choice of the alternative that appeared to provide one pellet involved the addition of one pellet. Although the reverse contingency might appear to be a difficult discrimination, in principle it combines an actual loss with an actual gain.

Importantly, Boysen and Berntson (1995) found that their chimpanzees had considerable trouble learning the reverse contingency task. Interestingly, however, the chimpanzees could accurately perform the reverse contingency task when the presented reinforcers were symbolically represented by Arabic numerals. Similarly, in our experiments, the color of each side of the apparatus provided a very salient conditioned stimulus.

\section{Procedure}

For half of the pigeons in Experiment 1 the color of the apparatus that displayed two pellets was the same as the color that displayed two pellets during the initial one- versus two-pellet testing. For the remaining pigeons, it was the other color.

In the reverse contingency procedure, each pigeon was presented with a choice between two pellets on one side and one pellet on the other. If the pigeon chose the two-pellet side, as it ate one of the pellets, the experimenter moved a slide over the second pellet on that side, preventing the pigeon from eating it. If it chose the one-pellet side, as it ate that pellet, a slide uncovered a second pellet. Thus, choice of two pellets provided one pellet, whereas choice of one pellet provided two. Each of six training sessions consisted of 32 trials.

\section{Results}

The pigeons readily learned to prefer the alternative that showed them one pellet but gave them two pellets over the alternative that showed them 2 pellets but gave them only one. The results of Experiment 1 are presented in Fig. 2. A repeated-measures analysis of variance performed on the data, with sessions as the repeated measure, indicated that there was significant learning of the alternative that gave them two pellets, $\mathrm{F}(5,35)=20.47, \mathrm{p}<.001, \mathrm{\eta}_{\mathrm{p}}{ }^{2}=0.75$. The proportion optimal choice on the last training session (Session 6) was .80. A single-sample t-test indicated that accuracy on the last session was significantly greater than $50 \%, \mathrm{t}(7)=5.63, \mathrm{p}<$ $.001,95 \% \mathrm{CI}=[.68, .93]$.

\section{Discussion}

The pigeons readily learned the reverse contingency task. It is very likely that color of the side of the apparatus together with

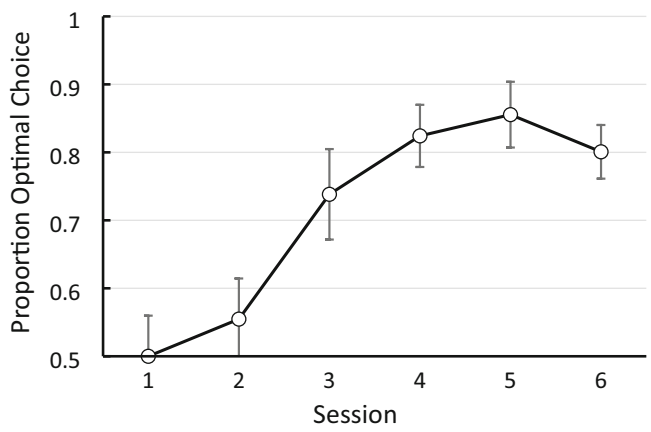

Fig. 2 Experiment 1: Reverse contingency experiment. If the pigeons chose the alternative that presented two pellets, they received only one pellet. If they chose the alternative that presented one pellet, they received an additional pellet. Error bars represent \pm 1 standard error of the mean 
the 5-s delay between baiting and access to the pellets facilitated acquisition, compared with chimpanzees. The social (competitive) context used in the Boysen and Berntson study may also have played a role in their failure to find consistent learning of the reverse contingency. However, we believe that the salient cues we used may have facilitated the discrimination in our experiment (as well as in Experiments 2 and 3). Furthermore, the 5-s delay between baiting and choice likely reduced impulsive choice (Zentall, 2020).

\section{Experiment 2}

The purpose of Experiment 2 was to separate the effect of the loss experienced after selecting the two-pellet alternative from the gain experienced after selecting the one-pellet alternative found in Experiment 1. In Experiment 2, each alternative displayed two pellets, but if the pigeon chose the two pellets on the "incorrect" color, one pellet would be removed.

To control for the color preference established in Experiment 1, for half of the pigeons the color assigned to the loss of a pellet was the same as their preferred color in Experiment 2. For the remaining pigeons, the color assigned to the loss of a pellet was the other color. On each trial, the pigeons were presented with two pellets on each side. If the pigeon selected the pellets on the "correct" color, it received the two pellets that were there. If the pigeon selected the pellets on the "incorrect" color, it received only one pellet (as it ate the first pellet, a slide covered the second pellet). In all other respects, the procedure was the same as in Experiment 1. The pigeons received 24 sessions of training.

\section{Results}

The pigeons readily learned to select the alternative that provided them with two pellets. The results of Experiment 2 are presented in Fig. 3. A repeated-measures analysis of variance performed on the data, with sessions as the repeated measure,

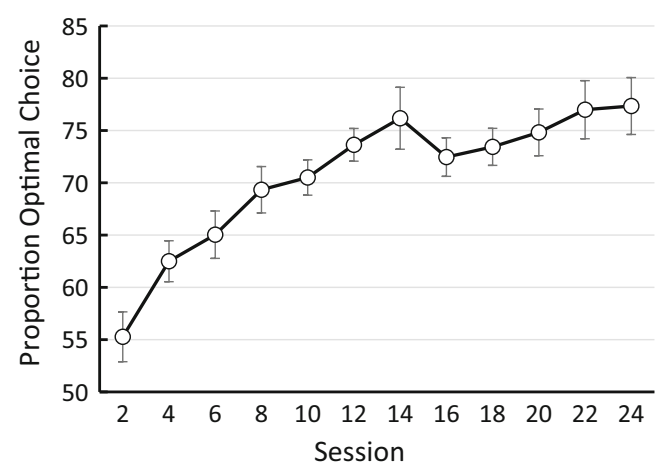

Fig. 3 Experiment 2: Pigeons were presented with two pellets on each alternative. If they chose the pellets on one color, one of the pellets was covered up. If they chose the pellets on the other color, they received both pellets. Error bars represent \pm 1 standard error of the mean indicated that there was significant learning of the alternative that gave them two pellets, $\mathrm{F}(23,161)=2.40, \mathrm{p}<.001, \mathrm{\eta}_{\mathrm{p}}{ }^{2}=$ 0.255 . The proportion optimal choice on the last two training sessions (Sessions 23 and 24) was .775. A single-sample t-test indicated that accuracy on the last two sessions was significantly greater than $50 \%, \mathrm{t}(7)=5.02, \mathrm{p}<.002,, 95 \% \mathrm{CI}=[.65$, .90].

\section{Discussion}

Acquisition of this task was somewhat slower than in the previous task because in this task the number of pellets presented could not be used as a cue to the alternative that would provide them with the greater magnitude of reinforcement. That is, the only cue provided was the color of the side that would provide them with two pellets. The pigeons had no difficulty learning to discriminate the color that provided them with two pellets.

\section{Experiment 3}

In Experiment 2, the correct choice by the pigeons resulted in one more pellet than the incorrect choice - an actual loss. The purpose of Experiment 3 was to test the hypothesis that pigeons would also prefer the absence of a loss over a perceived loss. In Experiment 3, the pigeons were presented with a choice of one pellet or two pellets. If they chose the onepellet alternative, they received that pellet. If they chose the two-pellet option, however, they received only one pellet because the second pellet would be removed. Thus, no matter which alternative they chose, they received one pellet. Three different outcomes of this experiment are interpretable. First, the pigeons presented with a choice of one pellet or two pellets could select the two-pellet option because the alternative that they could see had two pellets might be preferred over the alternative that they could see had only one pellet. Alternatively, because one pellet would be received in either case, the pigeons might be indifferent between the two options. Finally, when the pigeons choose the two-pellet option, they might find the loss of a pellet to be aversive, and they will learn to select the alternative that shows them one pellet, and reliably gives them one pellet. That is, they will show reference dependence in the form of loss aversion.

\section{Procedure}

Because the pigeons had strong color preferences at the end of Experiment 2, we assigned half of the pigeons to the twopellet condition with their preferred color from Experiment 2 and the remaining pigeons to the one-pellet condition with their preferred color from Experiment 2. All pigeons were shown two pellets on one alternative and one pellet on the 
other. If they chose the two-pellet alternative, a slide covered the second pellet. If they chose the one-pellet alternative they received the one pellet. Thus, regardless of their choice, they received one pellet. In all other respects the procedure was the same as in the earlier experiments. Experiment 3 consisted of 18 training sessions.

Silberberg et al. (2008) noted that in the Chen et al. (2006) study on loss aversion there was likely an added delay that resulted while removing one of the slices of apple. Silberberg et al. suggested that the added delay on the two-slice side could have accounted for the preference for the unchanged, one-slice reinforcer. With the use of the slides in the present experiments, there was no differential delay between access to the two alternatives because the slide covered the second pellet as the pigeon consumed the first pellet.

\section{Results}

Early in training, the pigeons showed a preference for the alternative that showed them two pellets but gave them only one (34.0\% choice of the one-pellet alternative on the first session of training). With continued training, however, the pigeons developed a strong preference for the alternative that showed them one pellet and gave them one pellet $(86.3 \%$ choice of the one-pellet alternative on Session 18). The results of Experiment 3 are presented in Fig. 4. A repeated-measures analysis of variance performed on the data with sessions as the repeated measure indicated that there was significant learning to choose the option in which only one pellet was offered, $\mathrm{F}(17,119)=10.46, \mathrm{p}<.001, \mathrm{\eta}_{\mathrm{p}}{ }^{2}=0.599$. The proportion optimal choice on the last two training sessions (Sessions 17 and 18) was .836. A single-sample t-test indicated that accuracy on the last two sessions was significantly greater than $50 \%, \mathrm{t}(7)=8.86, \mathrm{p}<.001,95 \% \mathrm{CI}=[.74, .93]$.

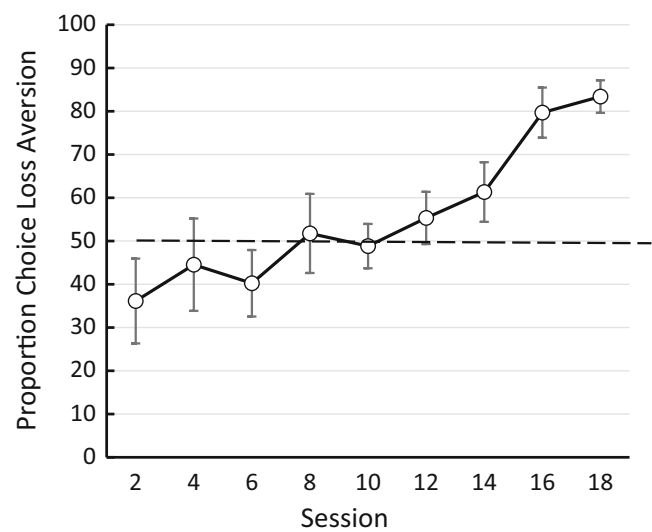

Fig. 4 Experiment 3: Reference dependence experiment. Pigeons were presented with a choice between two pellets and one pellet, but if they chose the two-pellet option, one of the pellets was removed. Thus, both options provided one pellet. Error bars represent \pm 1 standard error of the mean

\section{Discussion}

In Experiment 3, the pigeons showed a strong aversion to a loss. They preferred the alternative that showed them one pellet and gave them one pellet over the alternative that showed them two pellets but gave them only one. The loss of a pellet when two pellets are offered represents a loss that appears to lower the value of that alternative below that of the one-pellet alternative.

This finding indicates that it is not just the final outcome of the pigeons' choice that determines the pigeons' preference, but also the difference between what might have been expected and what actually occurred. This is a form of contrast that sometimes has been referred to as within-trial contrast (Case \& Zentall, 2018; Zentall, 2020). It is similar to what humans experience when expectations of outcomes fall short of what was expected (e.g., Kahneman \& Tversky's, 1979, prospect theory). It is also responsible for the sunk cost effect, the greater tendency that humans (Arkes \& Ayton, 1999) and other animals (Pattison, Zentall, \& Watanabe, 2012) have to persist in a behavior once an investment in money, effort, or time has been made. And it can explain the phenomenon found in humans called chasing losses (Lesieur, 1984), in which gamblers feel compelled to attempt to recoup experienced losses.

In the present experiment, one might speculate that, rather than an aversion to loss, the pigeons were averse to stimulus change. That is, the two-pellet alternative changes, whereas the one-pellet alternative does not. Recently, however, Clayton and Zentall (2021) conducted the reverse experiment (gain attraction), in which pigeons were offered a choice between two pellets and one pellet. If they chose the two-pellet option, they received the two pellets, but if they chose the onepellet option, they received an additional pellet. In this case, the pigeons developed a preference for the one-pellet option (which happened to involve a change). Thus, change avoidance was not likely responsible for the loss aversion effect found in Experiment 3. The results of the Clayton and Zentall experiment suggest that contrast between what was offered and what occurred was responsible for both the lossaversion and gain-attraction effects.

Although somewhat simpler than the procedure used by Chen et al. (2006) to study loss aversion in monkeys, the results of Experiment 3 were quite similar. That is, pigeons, much like monkeys (Chen et al., 2006), show reference dependence. They are averse to losing a piece of food even if the other alternative gives them the same amount of food. Reference dependence is a central principle in prospect theory (Kahneman \& Tversky, 1979). It holds that people evaluate outcomes and express preferences relative to an existing reference point. In the present experiment, one reference point is two pellets, and removal of one of the pellets makes that option worse. The other reference point is one pellet, but it does not get worse, so it is preferred. 


\section{General Discussion}

Reference dependence is related to the endowment (or mere ownership) effect. It is found when someone's willingness to pay to acquire an object is lower than the least amount they are willing to accept to give up that object when they own it even when there is no cause for attachment (e.g., they had no choice but were just given that object). The present results add to the growing literature that animals, as dissimilar from humans as pigeons, possess similar human biases (as well as cognitive abilities; Zentall, 2021).

Open practices statement The experiments reported in this article were not formally preregistered. Requests for the data or materials can be sent via email to the last author at zentall@uky.edu.

\section{References}

Arkes, H. R., \& Ayton, P. (1999). The sunk cost and Concord effects: Are humans less rational than lower animals? Psychological Bulletin, 125, 591-600.

Becker, G. S. (1962). Irrational behavior and economic theory. The Journal of Political Economy, 70, 1-13.

Boysen, S. T., \& Berntson, G. G. (1995). Responses to quantity: Perceptual vs. cognitive mechanisms in chimpanzees (Pan troglodytes). Journal of Experimental Psychology: Animal Behavior Processes, 21, 82-86.

Bush, R. R., \& Mosteller, F. (1951). A mathematical model for simple learning. Psychological Review, 58, 313-323

Case, J. P., \& Zentall, T. R. (2018). Suboptimal choice in pigeons: Does the predictive value of the conditioned reinforcer alone determine choice? Behavioural Processes, 157, 320-326.

Chen, M. K., Lakshminarayanan, V., \& Santos, L. R. (2006). How basic are behavioral biases? Evidence from capuchin monkey trading behavior. Journal of Political Economy, 114, 517-537.

Clayton, W. D., \& Zentall, T. R. (2021). Pigeons are attracted to a perceived gain without an actual gain. Animal Cognition.

Kagel, J. H., Battalio, R. C., Rachlin, H., Green, L. Basmann, R. L. \& Klemm, W. R (1975). Experimental studies of consumer demand behavior using laboratory animals. Economic Inquiry, 8, 22-38.
Kahneman, D., Knetsch, J. L., \& Thaler, R. H. (1990). Experimental Tests of the Endowment Effect and the Coase Theorem. Journal of Political Economy, 98, 1325-1348.

Kahneman, D., \& Tversky, A. (1979). Prospect theory: An analysis of decision under risk. Econometrica, 47, 263-91.

Lesieur, H. R. (1984). The chase: Career of the compulsive gambler. Cambridge, MA: Schenkman.

Mirenowicz, J., \& Schultz, W. (1994). Importance of unpredictability for reward responses in primate dopamine neurons. Journal of Neurophysiology, 72, 1024-1027.

Montague, P. R., Dayan, P., \& Sejnowski, T. J. (1996). A framework for mesencephalic dopamine systems based on predictive Hebbian learning. The Journal of Neuroscience, 16, 1936-1947.

Pattison, K. F., Zentall, T. R., \& Watanabe, S. (2012). Sunk cost: Pigeons (Columba livia) too show bias to complete a task rather than shift to another. Journal of Comparative Psychology, 126, 1-9.

Schultz, W., Dayan, P., \& Montague, P. R. (1997). A neural substrate of prediction and reward. Science, 275, 1593-1599.

Silberberg, A., Roma, P. G., Huntsberry, M. E., Warren-Boulton, F. R., Sakagami, T., Ruggiero, A. M., \& Suomi, S. J. (2008). On loss aversion in capuchin monkeys. Journal of the Experimental Analysis of Behavior, 89, 145-155.

Von Neumann, J. \& Morgenstern, O. (1944). Theory of games and economic behavior. Princeton, NJ: Princeton University Press.

Weaver, R., \& Fredrick, S. (2012). A reference price theory of the endowment effect. Journal of Marketing Research, 49, 696-707.

Zentall, T. R. (2020). Enhancing self-control: The paradoxical effect of delay of reinforcement. Learning \& Behavior, 48, 165-172.

Zentall, T. R. (2021). MacPhail revisited: Pigeons have much cognitive behavior in common with humans. Frontiers in Psychology: Section on Comparative Psychology.

Zentall, T. R., Case, J. P., \& Berry, J. R. (2017). Early commitment facilitates optimal choice by pigeons. Psychonomic Bulletin \& Review, 24, 957-963.

Zentall, T. R., \& Raley, O. L. (2019). Object permanence in the pigeon: Insertion of a delay prior to choice facilitates visible- and invisibledisplacement accuracy. Journal of Comparative Psychology, 133, 132-139.

Publisher's note Springer Nature remains neutral with regard to jurisdictional claims in published maps and institutional affiliations. 